\title{
Automated blood cell differential: State of the art and eye to the future ${ }^{1)}$
}

\section{Thomas Nebe ${ }^{1, *}$, Herrad Baurmann², Gudrun Kuling ${ }^{3}$ and Heinz Diem ${ }^{4}$}

${ }^{1}$ Laboratory for Special Haematology at Onkologikum

Frankfurt, Frankfurt/Main, Germany

${ }^{2}$ Centre for Bone Marrow and Blood Stem Cell

Transplantation, German Clinic for Diagnostics,

Wiesbaden, Germany

${ }^{3}$ Laboratory for Special Haematology of Robert Rössle

Klinik, HELIOS-Klinikum Berlin-Buch, Berlin, Germany

${ }^{4}$ Würmtal-Labor, Gauting, Germany

\begin{abstract}
Complete blood count including automated blood cell differential is one of the most frequent tests in the medical laboratory. How reliable is the method representing a diagnostic sieve in a stepwise diagnostic approach? Which pathologic conditions will be overlooked? Which body fluids can be investigated? What are the preanalytical requirements? When is a microscopic review required? Reimbursement has reached a level where a personal review of the diagram of cell clusters and of the blood film cannot be afforded. As the technology of blood cell analyzers has recently been described in this journal, this article focuses on the strengths and weaknesses of the automated blood cell differential. This allows judgment of the risks of a stepwise approach dependent on the medical discipline sending the sample.
\end{abstract}

Keywords: blood cell count; blood film review; flow cytometry; haematology; microscopy; platelet count; white cell count.

\section{Introduction}

Medical laboratories are currently undergoing major changes: As a result of rising cost pressures, small and

\footnotetext{
1)Original German online version at http://www.referenceglobal.com/doi/pdf/10.1515/JLM.2008.061.

The German article was translated by Compuscript Ltd. and authorized by the authors.

*Correspondence: Dr. med. Carl Thomas Nebe, Onkologikum Frankfurt am Museumsufer, Gartenstrasse 134, 60596 Frankfurt/Main, Germany

Tel.: +49 (069) 69-76-965-0

Fax: + 49 (069) 69-76-965-19

E-Mail: thomas.nebe@onkologikum-labor.de
}

medium sized clinical labs fall victim to cutbacks, but major clinics with their own labs, too, are experiencing staff shortages. The established labs are controlled by few private major laboratories with a high number of submissions, with daily analyses running from 5,000 to 10,000 blood cell counts. Haematological speciality labs at the clinic that have performed well for decades are now being shut down, with the samples being sent instead to a centralised lab that runs $500-1,000$ blood count tests every day. Control of special labs is increasingly handed to people who are not haematologists. Many medical technicians have no longer completed a specialisation in haematology, and in daily lab routines they show a lack of knowledge and experience. The pressure for more automation and equipment in haematology is growing, because microscopy is used less and less since morphology costs too much in terms of staff and time. At the same time there is an emergence of a few, inter-regional private special labs for haematology that specialise in the very diagnostic gaps left wide open by the major labs.

The introduction of an automated blood cell differential at the end of the 1980s was an important milestone in haematological diagnostics and was initially met with euphoria [1], however, its potential as a "diagnostic sieve" has been overestimated. These devices were developed to count and differentiate normal blood cells. Therefore, new equipment, too, shows a discrepancy between promotional messages in glossy brochures and actual performance in pathological samples. Thus, solutions must be found that can fill the current diagnostic gaps in automated haematological equipment in order to increase diagnostic accuracy in ruling out haematological diseases (see Figure 1). Algorithms can be established for a practical approach to keep sensitivity and specificity within reasonable limits (see Figure 2). Another critical aspect is the increasing use of blood count devices in diagnostic tests of body fluids and spinal fluid samples, both areas for which these devices were not developed and validated by the manufacturer.

\section{Erythrocytes}

The blood count is the most common lab test, with anaemia being the most frequent pathological result. While iron deficiency is one of the main causes, anaemia is 


\section{Haematological stage diagnostics}

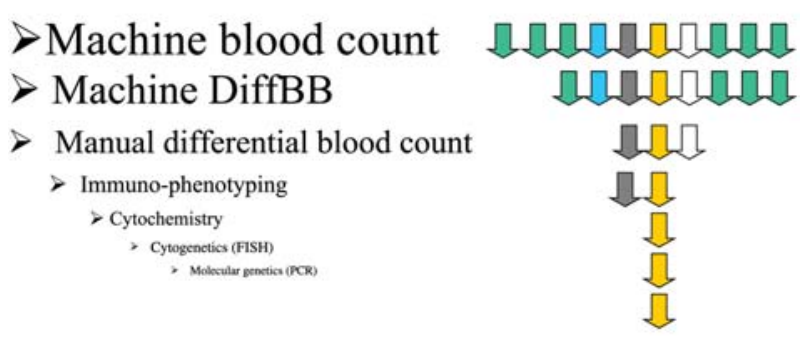

Diagnostic screen

Figure 1 Diagnostic screen.

Automated differential blood counts by way of a diagnostic screen occupies a key position in haematologic stage diagnostics, akin to the test strip and/or urine-flow cytometer in urine diagnostics.

caused by other factors in about $20 \%$ of these patients. For clarification purposes, there are various parameters that can be used, such as serum iron, transferrin saturation, ferritin and ferritin index (sTfR/log ferritin) including the actual C-reactive protein (CRP) value and the "Thomas Plot"-Reti-Hb versus sTfR/log ferritin, which must be utilised in a meaningful manner because the information obtained from a blood count is not sufficient. Zinc protoporphyrin in the erythrocytes - often ignored, but easily measurable using fluorometry - is a solid measurement that indicates iron-deficient erythropoiesis [2-4] and will be available in future haematology analyzers.

The insufficient basic knowledge in the diagnosis of anaemia manifests itself in daily lab requests where clarification of its cause is often incomplete or inconsequential. Since the determination of single parameters does not make much sense, entry profiles have been established that cover the most important causes of anaemia. In view of the low costs of basic diagnostics, a broader testing profile is recommended right from the beginning, which can then be supplemented during a follow-up.

The major haemoglobinopathies (thalassaemia, sicklecell anaemia, etc.) are not detected by haematological equipment up to now, despite their high incidence, even though certain constellations of $\mathrm{MCH}$ or MCV, RDW and red cell count would suggest the presence of such conditions.

The morphology of erythrocytes, crucial to differentiating the causes of anaemia, is captured only to a limited extent by current equipment that is used to measure erythrocyte volume and anisocytosis only. Changes in shape are not detected, resulting, for example, in target cells or fragmentocytes (which act as indicators for erythrocyte fragmentation syndromes) being overlooked. Even red cell inclusions like pyknotic nuclei in the case of the myelodysplastic syndrome (MDS), basophilic stippling in connection with toxic anaemia, Pappenheimer bodies or malaria parasites are not detected. Since a microscopic blood cell differential cannot be performed economically when it is priced at EUR 0.40, this test is often no longer provided and tends to be limited to leukocyte differentiation without any assessment of the red blood cells and thrombocytes.

All major equipment manufacturers today provide reticulocyte analysis. As the results of inter-laboratory experiments show, there are significant differences between the methods, which cause reference ranges and assessments to be dependent on the respective lab. Reduced reticulocyte counts can be detected only poorly by means of absorption dyes. The analysis of immature reticulocytes and the haemoglobin content of reticulocytes by fluorescent dyes with high affinity to ribonucleic acids (RNA) is helpful in providing early signs of success in substitution treatment [5]. The fact that the cost of this type of analysis is not covered by the German public health insurance anymore, will not lead to an increase of the clinical use and quality of laboratory analysis of reticulocytes.

Newer equipment provides the option of measuring nucleated erythrocytes [6-9]. The release of normoblasts is usually an important indication of the extent and success of treatment in the case of haemolytic anaemia. Most times, however, their frequency is lower than that of the leukocytes (1/10-1/100), which poses a problem when it comes to count statistics. More recent observations have identified the mobilisation of erythroblasts, as seen in disturbances of the blood-bone marrow barrier, as a sure sign of a bad prognosis for sepsis patients [10]. Furthermore, relatively high contents of normoblasts are found in umbilical cord blood and during the first few weeks after birth, where they have to be subtracted from the leukocytes. Attempts at validation in this area have yielded unsatisfactory results so far, since the cell counts determined manually are too low for sufficient statistics. Count accuracy in measuring these cell populations, at the relevant low concentration levels, is unsatisfactory and needs to be improved for all equipment tested so far. What is more, the crucial question of the morphology of the nuclei of erythrocytes cannot be answered yet by the equipment in terms of detecting important signs of dysplasia.

Therefore, any anaemia should be examined by an experienced individual at least once under the microscope as part of the initial diagnostic work up. The German Society of Haematology and Oncology has worked out a guideline for diagnosing anaemia, which can be accessed on the internet (http://www.dgho.de/ _cmsdata/_file/file_145.pdf).

\section{Thrombocytes}

Thrombocytopaenia in blood is not rare. The most important diagnosis to exclude a priori is the one of pseudothrombocytopaenia, i.e., an artificial in-vitro agglutination facilitated by IgM-antibodies that occurs mostly in EDTA 
blood, but on rare occasions also in citrated blood. In blood anticoagulated by heparin platelet agglutination is almost always present, which is why it is not suitable for blood count analyses. Under heparin therapy it may also occur in vivo and obtains clinical relevance. Another special case is the "satellite phenomenon" where thrombocytes attach to the surface of leukocytes. In essential thrombocythemia, a myeloproliferative condition, some patients also exhibit an aggregation of thrombocytes in vivo, which is also visible in smears and prevents a correct count. Here, too, there is the issue of a thrombotic risk. Counting thrombocytes in a haematocytometer by microscopy is a reference method for concentrations under 20/nL, where devices with impedance measurement experience problems. This manual procedure is not without problems and requires a certain level of experience. The count of low thrombocyte levels is technically difficult and can be improved substantially by using fluorochromated antibodies and flow cytometry [11].

As for citrate collection tubes, only those with a buffered citrate solution should be used all the time. As far as we know, the use of special collection receptacles such as ThromboExact has not yet been validated. The key differentiation with thrombocytopaenia is the distinction that must be made between reduced megakaryopoiesis (e.g., due to displacement) and distruction e.g., due to an antibody-triggered immunothrombocytopaenia with increased platelet production. If there is an increase in young thrombocytes, they can be stained - similar to reticulocytes as a subform of erythrocytes - using highaffinity RNA dyes. Therefore, they are called "reticulated thrombocytes", even though this is not morphologically accurate [12, 13]. Some nucleic-acid dyes, however, stain the granulomer of the thrombocytes partially unspecifically. The validation studies have not yet been concluded, since, unlike with reticulocytes, there is no microscopic reference method.

Idiopathic thrombocytopaenia (ITP) is often triggered by the antibody load on the platelets. The subform of the chronic immunothrombocytopaenia (M. Werlhof) is based on auto-antibodies against the gpllb/llla complex, which can be recognised reliably by special and complex tests (MAIPA, SASPA) [14, 15]. These tests, however, are only well established in larger transfusion departments of major clinics or blood donation services. But immunothrombocytopaenia can also be caused by paraneoplastic autoantibodies or immune complexes containing drug-related or infectious antigens. None of these is detected by the aforementioned tests and, thus, the platelet immunofluorescence test (PIFT) is still necessary. The poor specificity of these tests described in the literature [16] is due to the non-specific binding of serum immunoglobulins to Fc receptors, which have to be removed first, and the assay can be improved further by avoiding fixation.

In the case of ITP, complement mediated lysis creates thrombocyte fragments called platelet dust or platelet microparticles, which still promote coagulation. Patients with immunothrombocytopaenia, therefore, have a lower bleeding risk, despite an identical thrombocyte count, than patients with dyspoiesis of the thrombocytes [17, 18]. However, measurement of thrombocyte microparticles will only be available in future generations of haematology instruments.

In the event of disseminated intravascular coagulation (DIC), the drop in thrombocyte counts is one of the most sensitive parameters [19]. In healthy people the thrombocyte count is fairly constant and fluctuates a lot less than is suggested by the broad inter-individual reference range $(150,000-400,000 / \mu \mathrm{L})$. In this way, a drop is usually detected even before the thrombocyte count falls below the lower limit value. It is therefore crucial to conduct continuous previous-value comparisons. This socalled "delta check" can now be done by most of the laboratory information systems.

As for heparin therapy, the thrombocyte count must be checked in the same way in order to detect any drop in the thrombocyte count due to a heparin-induced thrombocytopaenia (HIT). An HIT type II is based on an immunological sensitisation and can occur after approximately one to two weeks after the initial heparinisation - and immediately if administered again. In the case of a known heparin therapy, heparin must be discontinued immediately before the results of the HIPA test are obtained (as a rule, no sooner than one to three days later).

The platelet aggregates can be detected pretty well by several analyzers using laser light scatter technology, while other large devices, which do basic blood counts in the first run only and giving their warning signals exclusively on the basis of volume measurements, are not sufficiently sensitive to detect this crucial interference.

The measurement of mean platelet volume (MPV) has still not reached any clinical significance. It cannot replace the analysis of reticulated thrombocytes in the case of ITP, and is only moderately sensitive to detect aggregates. Macrothrombocytes and megakaryocyte nuclei as indicators of myeloproliferative conditions are not detected as a population by any device.

A touchstone in terms of counting thrombocytes are erythrocyte fragmentation syndromes, such as the HELLP syndrome, Thrombotic Thrombocytopaenic Purpura or M. Moschkowitz, mechanical damage to erythrocytes (heart valve replacement, extracorporeal circulation, etc.) or the haemolytic uraemic syndrome (HUS). In these cases, thrombocytopaenia often occurs as a complication, which is why the small fragmentocytes must be separated effectively from the thrombocytes during the analysis - something that the mere volume measurement principle usually does not allow for. An optical analysis produces more reliable results, with the optimal method being the immunological count (antibody staining).

In summary, the same conclusion applies here as to the erythrocytes: In the initial examination, and when a deviation is first detected, a microscopic follow-up test will be necessary. 


\section{Leukocytes}

Measuring the number of leukocytes is a firmly established clinical parameter in the diagnosis of inflammatory conditions. As a rule, the equipment available in the market today can produce a normal diagnosis with a fivepart blood cell differential count based on fresh blood and healthy patients. After six to eight hours, however, the neutrophils in EDTA blood begin to disintegrate gradually (with considerable inter-individual variances), which can be shown particularly clearly using the light scattering analysis principle.

The neutrophil count as the most important criterion is detected reliably by the devices and is superior to the microscopic differentiation in patients with leucopenia. It should replace the leukocyte count due to its higher specificity in the diagnosis of inflammations. In the same way agranulocytosis would be detected sooner, because the leukocyte count in this condition does not drop below $1200 / \mu \mathrm{L}$ because of the still-present lymphocytes. But when it comes to the differentiation of bands (neutrophilic granulocytes with unsegmented nuclei) to describe the left shift, however, all manufacturers have failed thus far - except for the complex and rare image analysis systems. The reproducible count of bands also fails in the microscope review of blood films because of poor definition (segmentation rule with limit of thirds of nucleus diameter) and the bad counting statistics of 100 leukocytes when the same smear is presented to different people. The meaning of the analysis of bands has therefore been questioned by experts recently, and its usefulness is being debated in view of alternative inflammation parameters. The detection of more premature forms such as myelocytes and promyelocytes, however, is usually successful, at least, as a warning signal ("flag"), so that any pathological left shift should not be ignored or misinterpreted. Some instruments provide additional count results for precursor cells, but they cannot be verified microscopically. For validation purposes, immunological maturation parameters like CD11b and CD16 are utilised, which are more easily objectifiable.

Eosinophils show, apart from the brick-red colour as a reliable identification feature, depolarisation of polarised laser light. This measurement is interfered with only by the monocytes and granulocytes that have phagocytized malaria pigment, which occurs only rarely in our countries. They are slightly elevated with allergic conditions and substantially elevated with parasitoses, but only if the pathogen enters the tissue, for example, from the intestinal lumen. Other triggers, like paraneoplastic syndromes, are possible, too, which often makes clarification of the cause quite difficult. In the phase when patients overcome infections, there may be a slight and relative increase in eosinophils. A rarity is chronic eosinophilic leukaemia, which used to serve as a diagnosis of exclusion in the past [20]. Today, however, molecular markers are available [21]. A reduction in the eosinophil count is often a sign of an antiviral or bacterial immune response (lack of interleukin-5 from Th2 cells).

The reactive lymphocytes of a lymphocytosis described as virocytes or "Pfeiffer cells" are detected with moderate sensitivity by most devices (e.g., as large unstained cells (LUC) or blast cells). But the visible, activated, cytotoxic cells and their pseudopods are not detected but in the smear. Distinguishing blast cells of an acute leukaemia from the lymphoblasts of a virus infection is a task best left up to morphology and immunophenotyping. A microscopic assessment will be necessary at least to differentiate between a reactive and a malignant lymphocytosis. If it does not produce an unambiguous result, the method of choice becomes flow-cytometric immunophenotyping. The reactive increase of the lymphocyte count in connection with viral infections becomes less and less with age because of the existing memory cells and the involution of the thymus. Therefore, a lymphocytosis of over $4000 / \mu \mathrm{L}$ in older people must be seen primarily as potentially malignant. Conversely, many clonal lymphocytoses can be identified in this manner, whose clinical significance is still an open question.

Monocytes pose yet another problem area when it comes to automated blood cell differential counts. The variety in size and shape of these cells makes it difficult for morphologists and equipment developers to separate the small forms of the lymphocytes, or the large ones as macrophages, from granulocytes. Immunocytometry using fluorescent monoclonal antibodies usually utilises clones of the CD14 cluster, which, however, cover only approx. $85 \%$ of the mature monocytes and clones are subject to variances among manufacturers. Evaluation and validation studies of haematology equipment comparing devices using morphology and immunophenotyping help to illustrate this dilemma. Frequently an isolated increase of monocytes is a first sign of an infection with atypical pathogens (e.g., tuberculosis), endocarditis or haemolysis. Persistent monocytosis over $1500 / \mu \mathrm{L}$, however, can also be a sign of a myeloproliferative condition (MDS/MPD, CMMoL) in the absence of other clinical causes.

It is not rare for most of the instruments to incorrectly allocate other cells to the monocytes: Those cases are reactive lymphoblasts (e.g., EBV infection), plasma cells or immature blasts of an acute leukaemia. Therefore, a monocytosis diagnosed by means of a machine must always be verified by a review of the blood film. This poses a challenge, because these cells are mostly found at the end tip and along the edge of the smear, which attests to the inhomogeneity of the standard blood smear technique.

Basophilic granulocytes are and continue to be an issue by automated diagnostics, even though even a slight increase points relatively reliably to a myeloproliferative condition, apart from a few virus infections. Reality lags far behind the idealised depictions of colourful cell clouds in promotional brochures, particularly if the 
neutrophil content is reduced, e.g., in dysplastic changes.

\section{Leukaemia diagnostics}

One of the most important warnings in haematology equipment is the "blast alert". Undifferentiated blasts can be detected on the basis of the properties of scattered light or, better yet, cytochemical properties. Sensitivity, however, is usually limited as far as the minimum number of mobilised blasts and the various types of leukaemia are concerned. Small blasts of a common ALL or T-ALL are often found in the area of the normal lymphocytes and are not detected. Thus, excluding circulating blasts reliably still requires microscopic testing and immunophenotyping. The new image analysis systems for clinical use, too, leave a lot to be desired. If a lower blast count is suspected or expected (MDS, CMPE or minimal residual disease (MRD) following ALL/AML) microscopic testing and immunophenotyping must be used right from the beginning. In many laboratories, the warning threshold for blasts of the instrument has been reduced to lower the number of false positive alerts and subsequent postdifferentiations to a reasonable level.

The most common adult-age form of leukaemia is chronic lymphocytic leukaemia (CLL). Unlike acute leukaemia, it often shows no signs of anaemia or thrombocytopaenia. In many cases, the small lymphocytes are counted without triggering any alert. There are, however, other low-malignant B-Non-Hodgkin lymphomas with spreading into peripheral blood, thus necessitating at least microscopy and immunophenotyping to arrive at a reliable diagnosis. The shadows in the smear of CLL are biologically aged, fragile lymphocytes that are not detected by some devices - due to their measuring techniques and lysis reagent. Extrapolating the number of leukocytes by analysing lysis kinetics produces serious errors in practice.

Myelodysplastic Syndrome (MDS) tends to occur with advancing age as anaemia that does not respond to any kind of therapy and is often the precursor of an acute, mostly myeloid, leukaemia. The signs of dysplasia from neutrophils or erythroblasts in blood smears may raise suspicion, but in many cases, they are only detected by experienced staff during bone marrow cytology. Secondary leukaemia resulting from MDS often exhibits only a low level of circulating blasts, i.e., a normal leukocyte count. This poses a special challenge that underlines the crucial significance of careful microscopic testing of blood smears stained according to Pappenheim. A diminished side scatter light signal of neutrophilic granulocytes can occur with both toxic granulation in the case of infections and diminished granulation in connection with MDS and is, therefore, not MDS-specific [22].

Leukaemia diagnostics requires its own stepwise diagnosis and cannot be limited only to an immunological allocation of blast crises to the myeloid or lymphocytic series with few markers on fully automated equipment. The analysis of bone marrow, biopsies and lymph nodes with the methods of cytology, cytochemistry, enzyme immunology, immunophenotyping and molecular genetics yields parts of a puzzle that have to be assembled in full in order to arrive at a diagnosis and prognosis. The detection of minimal residual disease (MRD diagnostics) is a special case that is currently being studied and economically useful for only few laboratories. The topic of leukaemia diagnostics has already been dealt with in detail, which is why this paper will forego any further analysis [23-26].

\section{Stem and progenitor cells}

In clinical practice flow cytometry of CD34-positive cells is used to monitor the point of leukapheresis in patients prior to autologous bone marrow transplants or to clarify the diagnosis of aplastic anaemia. Another use includes clarification of ambiguous findings regarding circulating blasts. But this carries a risk, because several forms of acute leukaemia do not express CD34, nor do blastic lymphomas. This means that these parameters on their own will not rule out these diagnoses even when few other markers are added (e.g., CD117). The detection of progenitor cells by automated haematology equipment as a population of immature cells with abnormal physical-chemical parameters, such as scattered light and resistance measurement, is unreliable even if in special situations (point of leukapheresis in patients following stem-cell mobilisation) there is a certain correlation with the correct immunophenotyping method [27].

\section{Malaria diagnostics}

The accidental discovery of the depolarising property of phagocytosed malaria pigment in monocytes and its detection by a laser-based haematology analyzer in a laboratory in South Africa attracted a lot of attention [28]. On closer inspection however, and based on own experience, this method is not sufficiently sensitive to replace the traditional methods of blood smear tests or thick blood films [29]. This method, therefore, is not suitable for screening tests. Furthermore, the significance of phagocytosed malaria pigment is considered controversial with respect to prognosis. The use of other parameters like monocyte volume is obsolete, although affected patients, on average, exhibit values that deviated from the norm. Immunological antigen tests on test strips (histidine-rich protein and aldolase) have become reliable tools in the hands of experienced staff to screen for malaria. The intensity of infestation and type of plasmodium, however, must still be determined by microscopy; the same is true for a diagnosis of a mixed infection. A repeated and thorough screening of the blood smear established by the laboratory at a different time is seen 
as sufficient even by experts and thus renders the dreaded thick blood film in a routine laboratory unnecessary.

\section{Analysis of body fluids}

Analysing the cell count and $\mathrm{Hb}$ content in pleura and joint fluid aspirates using automated haematology equipment and EDTA samples is a common practice, even though the equipment is usually not licensed for such use by manufacturers. In the case of purulent discharge, leukocytes (granulocytes) disintegrate, and cell concentration is massively under- or overestimated, depending on the measuring parameters used. The differentiation of leukocytes in those fluids is successful only with few devices and should definitely not replace microscopy; doing so would result in bacteria or tumour cells being overlooked. Still, manufacturers are pushing into this market, because staff shortages at laboratories necessitate alternatives [30].

\section{Diagnostics in cerebrospinal fluid (CSF)}

Determining the cell count under the microscope in the Fuchs-Rosenthal chamber, a special form of a haematocytometer, is difficult and time-consuming. What is more, the cells in the spinal fluid do not keep very long ( $\ll 2 \mathrm{~h}$ ), except when there is a substantially elevated protein level. In stained microscopic cytospin slides, these dead cells appear as faint nuclear shadows. Spinal fluid samples must therefore be counted immediately after winning. In view of staff shortages, there is a strong tendency at labs towards running the samples through haematology devices [31-33]. Once this is established, it will not be long before the differentiation determined by the device is accepted as accurate so as to avoid having to prepare and analyse a stained cytospin slide at night. This comes at a great risk: Only few devices are capable of reaching the count limit for the standard range $(<5$ leukocytes $/ \mu \mathrm{L}$ ), because the equipment has been designed for whole-blood analyses, but the sample volumes and counts are simply too low. In the range of 20-50 cells, the cell count is overestimated by most of the instruments. All these attempts require that the equipment be cleaned and rinsed thoroughly prior to analysis in order to lower the blank value due to contamination. On the other hand, the devices provide valuable assistance in evaluating the cell count of bloody spinal fluid, where the staining solution cannot lyse the erythrocytes. The light scatter properties of the cells in the spinal fluid is so different for macrophages, in particular, from those in the blood, for which these devices have been designed, that the cells in the CSF cannot be assigned reliably. There are new haematology devices on the market today that have been improved for this type of use (higher volume, adaptation of lysis and software). However, bacteria and fungi are still not detected, which is why low-cell, bacterial meningitides (often with ventricular spinal fluid from the catheter from ICU patients) or fungal meningitis (e.g., cryptococcal meningitis in connection with HIV infection) go unnoticed without microscopy. An uncontrolled, or even exclusive, use of haematology equipment is therefore irresponsible in connection with cerebrospinal fluid testing.

\section{Developments in the measuring technique of haematology equipment}

The historical basic procedure is impedance measurement, i.e., changes in the resistance of an electrolyte (isotonic saline solution) at the time a cell passes through a measurement aperture. It serves as a counting impulse, while the height or integral of the impulse acts as a measure of the cell's size. This method is sufficient for the complete blood count of a healthy individual, but not for pathological blood counts (overlapping of distribution curves). The next stage in the development, the introduction of high-frequency resistance measurement in addition to direct-current resistance measurement, did not produce any breakthrough. Next came the use of lasers for light scattering analysis at different angles to the incident laser beam. All parameters, including the blood cell differential count, have substantially taken advantage from this. The detection ranges were expanded, and the separation of cell populations and measuring speed improved. This concerns, for example, the problematic resolution of thrombocytes, fragmentocytes, microcytic erythrocytes, or improved alerts for thrombocyte aggregates or granulocytic precursor cells. In addition, the use of a laser and the less expensive laser diodes also allows for fluorescence dyes to stain cell types differently. This concerns reticulocytes as well as the separation of erythrocytes, macrothrombocytes, nucleated erythrocytes from leukocytes.

Not in normal samples but in a substantial number of pathological samples, the equipment or analytical software cannot produce reliable results for thrombocytes or determine the white cell differential. Universal problems in the current generation of modern haematology devices include the aforementioned separation of monocytes and basophils from lymphocytes, the detection of blast cells or of bands or metamyelocytes within the left shift. A possible solution is the use of immunofluorescence. For this purpose, cell-specific surface proteins are stained with fluorochrome-conjugated antibodies. As a result of the multi-colour fluorescence, a reliable blood cell differential can be established, including hints for the presence of acute leukaemia or lymphoma.

Working parties of laboratory haematologists in Europe and the U.S. are currently studying which antibodies are best suited for immunological blood cell differential and which weaknesses of the current method can be solved in the most efficient way. The first publications on the topic, by Teerstappen, date back to the end of the 1980s 
[34]. The costs, however, are a multiple of the costs for a standard automated blood cell differential.

\section{Software for haematology equipment}

Progress has been made in this area as far as userfriendliness, validation, measured results and quality control are concerned. However, the quality of measured results can be improved by software changes only to a limited degree. The resolution and separation of the cell clusters in multi-parameter measurements has been improved, and their locations (position of the cloud in the $X Y$ diagram) can indicate abnormal conditions, but the rate of necessary subsequent microscopic differentiation has not been lowered substantially. For the respective laboratory, it is constantly in the range of $10 \%-30 \%$, depending on the basis of patients. The comparison with previous values of the patient plays a vital role in haematology, e.g., in terms of plausibility control, excluding any confusion of samples, or the drop of haemoglobin or thrombocytes as sensitive parameters of consumption coagulopathy or heparin-induced thrombocytopaenia. The hand-written notes attached to the analyzer with warnings about special problem patients (e.g., cryoagglutinins, EDTA-pseudothrombocytopaenia) for the next shift should really be a thing of the past today. Oftentimes hospitals and large labs operate several instruments in parallel. Regardless of whether the analysers are from the same or different manufacturers, they produce results that differ more or less systematically. As they have to be harmonised with each other, thus creating considerable expenditure for the lab. Manufacturers still provide insufficient support as concerns the software. When the throughput rate is high, many samples and only few controls are measured. As a result, the continuous monitoring of the mean value (X-B algorithm) is a proven method to determine a drift of the equipment early on. Unfortunately, this so-called "bull algorithm" is still available only for few parameters (mostly only for the $\mathrm{Hb}$ value) and devices.

\section{Lymphocyte typing}

Immunophenotyping of lymphocyte subpopulations in peripheral blood using fluorescent monoclonal antibodies and flow cytometry is a not yet fully automated method that has become established practice in most larger specialised laboratories. Hard indications for this analysis are very limited, like monitoring for HIV infection for serious therapeutic immunosuppression or clarifying ambiguous blood counts in connection with lymphocytosis or suspected leukaemia. The significance for other immunodeficiencies is low, apart from children with suspected congenital cellular immunodeficiency, which is in stark contrast to the frequent submissions that ask for this test. The usually supplied standard parameters T-, B-, NK cells with CD4 and CD8 lymphocytes should be supplemented to include a light chain restriction analysis of the B cells (kappa/lambda), because B-NHL is the most common cause of malignant lymphocytosis. These analyses might be available in an automated fashion in haematology analyzers using lasers with blue or violet excitation. Any further immunophenotyping should be handled by specialised laboratories only that have the necessary morphological expertise and a more broadbased testing programme, thus enabling them to arrive at a definitive diagnosis most of the time. Immunomonitoring or the cellular immune status only makes sense when placed in the context of a specific clinical problem; its interpretation is only feasible when the clinical history of the patient is taken into account. Only few large clinics with specialised hospital departments have been shown to be capable of doing that.

The considerable increase of the available repertoire of fluorescent monoclonal antibodies against human leukocyte antigens (CD nomenclature with almost 400 antibodies) has so far yielded no major progress in the number of diagnosable conditions - apart from few exceptions, such as the rare paroxysmal nocturnal haemoglobinuria $(\mathrm{PNH})$.

\section{New and innovative parameters}

When looking at turnaround times, from the taking of a sample to the result, the blood count is by far the fastest laboratory parameter, since the sample does not have to be centrifuged, and because incubation and measurement times are very short. By measuring soluble analytes on antibody-coated fluorescent microparticles (suspension bead array technique), it will be possible, in future, to analyse vital analytes such as troponins simultaneously with the blood count from whole blood. As well, the fluorescence properties of normal cells, such as the autofluorescence of zinc protoporphyrin in iron-deficient erythrocytes, can produce substantial advances in the diagnosis of iron deficiency anaemia when integrated with laser based haematology analyzers.

\section{Preanalytics and blood ageing}

Preanalytical considerations represent an unpopular topic in medical lab diagnostics, since that part is controlled by submitters and transport routes, with the laboratories having only limited influence. In haematology, however, it seriously affects the quality of results and, therefore, plays an important role. Skilled specialised laboratories have therefore moved to providing special transport containers and shipping modes. Still, the blood smear should be done immediately after the drawing of blood, with the unstained slide being sent to the lab.

The anticoagulant of choice for blood counts is still EDTA, with slight variations for K2 and K3 EDTA. The 
analysis has to be done after a reaction time of at least $15 \mathrm{~min}$ so as to obtain correct MCV values. When the sample is not mixed right away or is refilled from another sampling tube (obsolete), small thrombocyte aggregates are formed that are visible in the smear even before the device can issue a warning. Even when the sample has coagulated fully (clotting) no flag is coming up. In terms of the speed of anticoagulation, EDTA is superior to other anticoagulants. Heparin disturbs in several ways (platelet aggregation, cell activation with vacuole formation, coloured precipitate), and liquid anticoagulants (citrate) lead to dilution and incalculable cell concentrations if the sampling tube is not filled completely.

The morphology of the cells in EDTA blood, depending on the individual; keeps only four to eight hours which has a great influence on the interpretation of the smear. Neutrophilic granulocytes and monocytes then get vacuolisation, which is no longer the result of an activation in vivo. Lymphocytes develop apoptotic forms, which cannot be attributed to a viral infection or which do not allow for a differentiation of lymphocytic neoplasias. In the course of time, neutrophils are affected by nucleus fragmentation, which makes them fall below the count threshold of the devices. Stability studies that show a considerable longer useful storage time of EDTA blood are mostly limited to parameters of the small blood count and blood samples from healthy donors [35].

The sensitivity in terms of blood ageing is depending on the measuring technique: For example, optical light scattering measurements alone and an aggressive lysis method for erythrocytes prevent the neutrophils from being measured correctly the following day.

In any case, stability depends on the instrument and shipping method employed and is, thus, specific to each laboratory, which is why the inspection of the sample upon receipt is among the knock-out criteria in laboratory accreditiation. Temperature-stable shipping at $10-14^{\circ} \mathrm{C}$ allows for transport within max. $24 \mathrm{~h}$. However, no method achieves $48 \mathrm{~h}$ preservation for neutrophils. The evaluation of temperature loggers in the transport container for the purposes of validating inter-laboratory tests often comes with surprises, e.g., when air transport or frost periods are involved. Stabilisation media, either fixing or non-fixing, have been successful so far only with measuring certain immunological properties of lymphocytes under critical testing. New and improved anticoagulants have not found their way into routine laboratories yet.

\section{Blood samples from children}

Blood samples from babies and infants pose a special challenge and have been neglected by manufacturers in the development of instruments. The available blood volume in the case of capillary blood samples is below the minimum suction volume of large equipment for the fivepart blood cell count of approximately $150 \mu \mathrm{L}$. In newborns, the percentage of nucleated red blood cells is high. Devices working with small volumes $(<50 \mu \mathrm{L})$ so far count them as leukocytes or issue a warning flag at best.

The most common method used in the laboratory is to manually predilute such samples $(1: 3-1: 5)$ and to run them on their standard haematology device with blood cell differential. This predilution method has been validated, if at all, on the basis of blood taken from healthy adults. If such measurements are compared for children where alternately diluted and undiluted samples were analysed, values usually fluctuate considerably. Furthermore, predilution can even cause false results in some devices $(\mathrm{Hb}, \mathrm{MCV})$.

Since the liver of premature babies is not developed yet, the level of acute-phase proteins in the serum is not suitable for diagnosing inflammation. Therefore, in paediatrics, the immaturity or left shift, i.e., the ratio of immature to total neutrophils ("I/T ratio"), is used instead of the C-reactive protein (CRP). All attempts to replace this value by surrogate markers of the haematology analyzer have to be regarded as having failed, because banded neutrophils, which contribute substantially to the shift of the ratio, cannot be detected separately by the devices. The immunologically measured left shift via the expression of the low or high-affinity receptor for IgG (CD16 or CD64) represent expensive alternatives, which must also be validated and are not yet suitable for routine testing in emergency laboratories. There have not been any comparative studies on procalcitonin yet either.

\section{Standard ranges}

When it comes to the complete blood count, it is possible to define reference ranges across devices as well as specific to age and gender. For the reasons already stated, however, one must expect substantial differences with respect to reticulocytes and the blood cell differential, particularly in connection with monocytes and basophilic granulocytes. The results of a multi-centre study by DGHO and INSTAND on this topic are not yet available.

\section{Warning notes}

The concept of haematology devices has been developed for the reliable detection of normal blood cells. Abnormal samples and cell types are available to the development laboratories of the manufacturers only in very limited numbers. Consequently, the devices' practical suitability can be assessed only when challenged with pathological samples as part of clinical trials. However, by that time, the design and construction phase has already been finished. There are only very limited chances for correcting the measuring technique at such time, with the corrections then usually ending up being software adjustments, if that. What is more, the warning notes (so-called "flags") vary from one device type to the 
next due to the differences in technology and software. Some devices can detect interferences quite well (e.g., thrombocyte aggregates, macrothrombocytes, normoblasts), while other instruments even from the newest generation fail. Their possibilities in the detection of abnormal cells are limited, also in the case of blasts. Neutrophils with band shaped nuclei (also known as bands) are not recognised by any device. Instead, it comes down to a rough suspicion based on cell size and neutrophil count $(>10-15,000 / \mu \mathrm{L})$.

The warning limits of an analyzer can be adjusted in the software, which affects sensitivity and specificity of a flag considerably. On one hand it is not helpful when one out of three samples triggers a blast alert, but on the other it is bad, when $50 \%$ of the samples with a low blast content do not give an alert. Large laboratories tend to lower the sensitivity level in order to minimise the postdifferentiation rate. In these instances, technicians with little haematological experience would not have much chance anyhow trying to classify pathological cells under a microscope. In specialised laboratories with a small number of samples and a high percentage of pathological samples, however, clusters on data plots of any patient can be validated manually and pathological images recognised quickly. Complex rule systems in downstream data stations, which take into account the type of sender (i.e., the prevalence of certain conditions), can improve the situation only to a limited extent. An international group of experts has agreed on general review criteria for blood counts that are supposed to be independent of the device [36], but overall, such rules cannot replace the quality of a high-resolution measurement of normal and pathological cell populations with several independent parameters.

\section{Alert constellations}

Despite the aforementioned limits, there are characteristic diagnostic constellations with respect to the automated blood count that require immediate clarification by a haematologist and are part of the review criteria referenced:

1. Anaemia ( $\mathrm{Hb}<110 \mathrm{~g} / \mathrm{L}$ (male) or $100 \mathrm{~g} / \mathrm{L}$ (female)) and thrombocytopaenia $(<120 / \mathrm{hL})$ : Suspected impairment of haemaopoiesis requires bone marrow biopsy

2. Leucopenia with anaemia, thrombocytopaenia and reduction of reticulocytes: Suspected aplastic anaemia, MDS...

3. Leukocytosis with anaemia and thrombocytopaenia: Suspected sepsis, acute leukaemia...

4. Isolated lymphocytosis ( $>3500 / \mu L$ in adults): Suspected EBV, CLL, NHL...

5. Anaemia with reticulocytosis and normoblasts: Suspected haemolysis

6. Fragmented red cells ("helmet cells") in the smear $(>1 \%)$ : Suspected erythrocyte fragmentation syndromes, such as HELLP, TTP, HUS... (see above)

7. Isolated neutropaenia $(<500 / \mu L)$ : Suspected acute viral infection, agranulocytosis, sepsis...

8. Isolated monocytosis $(>1500 / \mu \mathrm{L})$ : Suspected CMMoL, CMPE... (see above)

9. Drop in the thrombocyte count $(>30 \%$, sample not clotted): Consumption coagulopathy, heparininduced thrombocytopaenia

\section{Stepwise diagnostics}

It is quite self-evident that a stepwise diagnostic diagram depends on the question to be addressed and the prevalence of the condition to be ruled out or confirmed. Prevalence affects the diagnostic sensitivity and specificity of every test significantly (predictive value). On the other hand, there are the diagnostic skills of the laboratory and its know-how.

Having this in mind, several situations can be defined. On the part of the submitting doctor:

- Situation 1 is the general practioner.

- Situation 2 is the practising consultant (internal medicine).

- Situation 3 is the practising haematologist or the haematological department of a hospital.

On the part of the laboratory, there is:

- Situation A of a large basic laboratory.

- Situation B of a large laboratory for special analyses.

- Situation C of a specialised haematological laboratory with its separate management, proven haematological expertise and experience and continuous professional development.

From the existing situation described above considerable conclusions have to be drawn and thus, specific diagnostic programmes as haematology is concerned can be defined:

1. Basic diagnostics:

a. for general health check: complete blood count w/ o differential

b. persistent fatigue, weakness: blood cell differential in addition, if necessary virus serology

c. recurring infections: blood cell differential including smear

2. Extended diagnostics in case of pathological finding or case record: In case of...

a. anaemia: blood cell differential, smear and anaemia diagnostics (see guidelines)

b. leukocytosis: smear and if necessary immunophenotyping

c. leucopenia: smear and if necessary immunophenotyping 


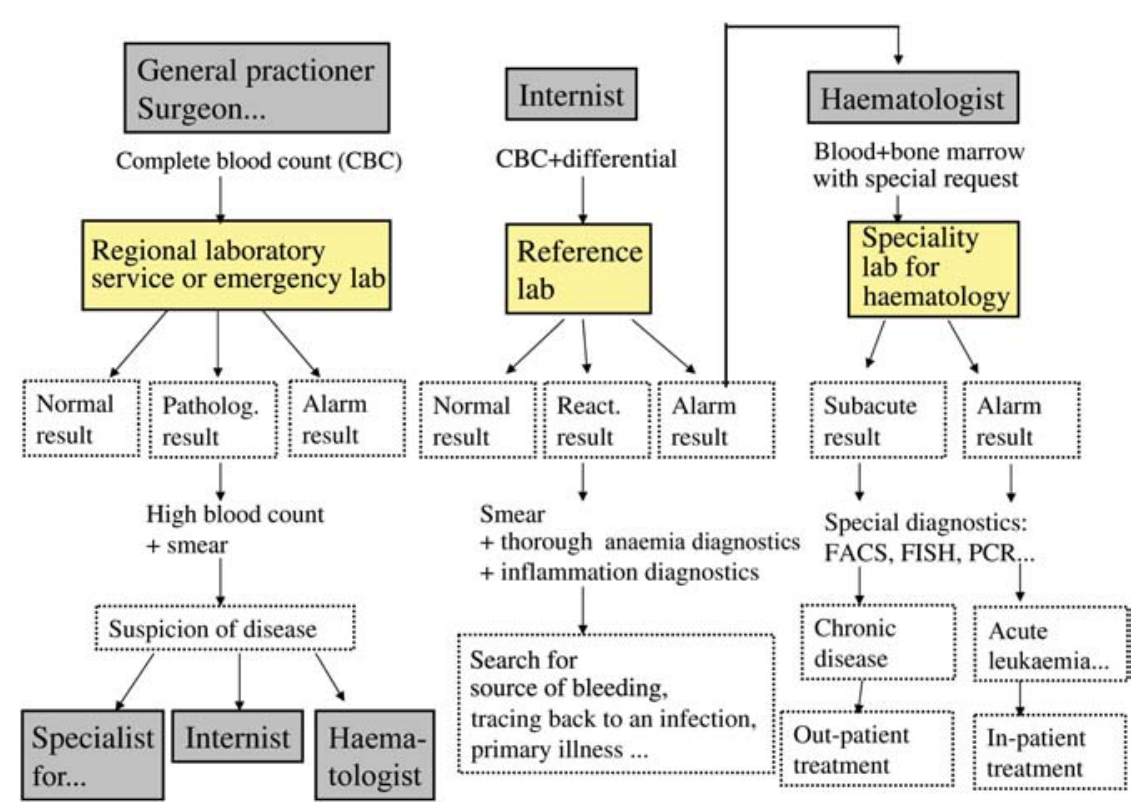

Figure 2 Stage diagnostics and sender.

The significance of a blood count varies depending on the sender, and is produced by different laboratories. Furthermore, the special laboratories are necessary for haematologists who leave the clinic for private practice.

d. thrombocytosis: smear and diagnostics of inflammations including autoimmune diseases

e. thrombocytopaenia: smear, reticulated platelets and if necessary detection of platelet auto-antibodies in case of non-reactive changes

From this, the following flow chart can be developed (Figure 2).

\section{Conclusion}

The automated blood cell differential count is one of the most common laboratory tests that takes up a central role in today's haematological diagnostics, in the form of a diagnostic sieve. This has been accounted for by the recent introduction of external quality assessment for these tests. When it comes to clarifying pathological findings, the devices currently available on the market do not meet the requirements when used exclusively. The microscopic review of the stained blood film by well trained specialists continues to be an indispensable tool and ought to be demanded by the submitting physician, because this type of test, given its poor reimbursement, is handled in a restrictive manner at this level, which also limits its quality. Nevertheless, the microscopic blood cell differential will be replaced by immunophenotyping in years to come due to its automated aspect and cost reimbursement. The expensive dedicated manual diagnostics such as immunophenotyping, PCR, etc. used to clarify immunodeficiencies or systemic haematological disorders must be left up to specialists, both in clinical and diagnostic terms; otherwise, there would be false interpretations and repeated analyses. The increasing exclusive utilisation of blood count devices for count and differentiation of cells in body fluids even with improved software is risky for the patient, because crystals, bacteria, fungi and tumour cells and, thus, important clinical pictures will be overlooked.

\section{References}

1. Krause JR. Automated differentials in the hematology laboratory. Am J Clin Pathol 1990;93:S11-6.

2. Thomas C, Thomas L. Anemia of chronic disease: pathophysiology and laboratory diagnosis. Lab Hematol 2005;11: 14-23.

3. Hastka J, Lasserre JJ, Schwarzbeck A, Hehlmann R. Central role of zinc protoporphyrin in staging iron deficiency. Clin Chem 1994;40:768-73.

4. Metzgeroth G, Hastka J. Diagnostic work-up of iron deficiency. J Lab Med 2004;28:391-9.

5. Thomas C, Kirschbaum A, Boehm D, Thomas L. The diagnostic plot: a concept for identifying different states of iron deficiency and monitoring the response to epoetin therapy. Med Oncol 2006;23:23-36.

6. Kim YR, Yee M, Metha S, Chupp V, Kendall R, Scott CS. Simultaneous differentiation and quantitation of erythroblasts and white blood cells on a high throughput clinical haematology analyser. Clin Lab Haematol 1998;20:21-9.

7. Tsuji T, Sakata T, Hamaguchi Y, Wang F, Houwen B. New rapid flow cytometric method for the enumeration of nucleated red blood cells. Cytometry 1999;37:291-301.

8. Briggs C, Harrison P, Grant D, Staves J, MacHin SJ. New quantitative parameters on a recently introduced automated blood cell counter - the XE 2100. Clin Lab Haematol 2000; 22:345-50. 
9. Igout J, Fretigny M, Vasse M, Callat MP, Silva M, Willemont $\mathrm{L}$, et al. Evaluation of the coulter LH 750 haematology analyzer compared with flow cytometry as the reference method for WBC, platelet and nucleated RBC count. Clin Lab Haematol 2004;26:1-7.

10. Stachon A, Holland-Letz T, Krieg M. High in-hospital mortality of intensive care patients with nucleated red blood cells in blood. Clin Chem Lab Med 2004;42:933-8.

11. Kunz D. Possibilities and limitations of automated platelet counting procedures in the thrombocytopenic range. Semin Thromb Hemost 2001;27:229-35.

12. Ault KA, Rinder HM, Mitchell J, Carmody MB, Vary CP, Hillman RS. The significance of platelets with increased RNA content (reticulated platelets). A measure of the rate of thrombopoiesis. Am J Clin Pathol 1992;98:637-46.

13. Briggs C, Kunka S, Hart D, Oguni S, Machin SJ. Assessment of an immature platelet fraction (IPF) in peripheral thrombocytopenia. Br J Haematol 2004;126:93-9.

14. Kiefel V. The MAIPA assay and its applications in immunohaematology. Transfus Med 1992;2:181-8.

15. Nguyen XD, Dugrillon A, Beck C, Kerowgan M, Kluter H. A novel method for simultaneous analysis of specific platelet antibodies: SASPA. Br J Haematol 2004;127:552-60.

16. Nishioka T, Yamane T, Takubo T, Ohta K, Park K, Hino M. Detection of various platelet-associated immunoglobulins by flow cytometry in idiopathic thrombocytopenic purpura. Cytometry B Clin Cytom 2005;68:37-42.

17. Jy W, Horstman LL, Arce M, Ahn YS. Clinical significance of platelet microparticles in autoimmune thrombocytopenias. J Lab Clin Med 1992;119:334-45.

18. Piccin A, Murphy WG, Smith OP. Circulating microparticles: pathophysiology and clinical implications. Blood Rev 2007; 21:157-71.

19. Vanderschueren S, De Weerdt A, Malbrain M, Vankersschaever D, Frans E, Wilmer A, et al. Thrombocytopenia and prognosis in intensive care. Crit Care Med 2000;28:1871-6.

20. Simon D, Simon HU. Eosinophilic disorders. J Allergy Clin Immunol 2007;119:1291-300.

21. Fletcher S, Bain B. Diagnosis and treatment of hypereosinophilic syndromes. Curr Opin Hematol 2007;14:37-42.

22. Valent P, Horny HP, Bennett JM, Fonatsch C, Germing U, Greenberg P, et al. Definitions and standards in the diagnosis and treatment of the myelodysplastic syndromes: Consensus statements and report from a working conference. Leuk Res 2007;31:727-36.

23. Szczepanski T, van der Velden VH, van Dongen JJ. Flowcytometric immunophenotyping of normal and malignant Iymphocytes. Clin Chem Lab Med 2006;44:775-96.
24. Bettelheim P, Diem H, Nebe T. Durchflusszytometrische Typisierung der B-Zell-Non-Hodgkin-Lymphome. J Lab Med 2004;28:410-23.

25. Schoch C, Kern W, Schnittger S, Haferlach T. FISH-Diagnostik bei hämatologischen Neoplasien. J Lab Med 2005; 29:335-42.

26. Kern W, Schnittger S. Monitoring of minimal residual disease in acute myeloid leukemia. J Lab Med 2005;29:34367.

27. Oelschlaegel U, Bornhaeuser M, Thiede C, Ehninger G, Hoelig K. HPC enumeration with the Sysmex XE-2100 can guide further flow cytometric CD34(+) measurements and timing of leukaphereses. Cytotherapy 2003;5:414-9.

28. Mendelow BV, Lyons C, Nhlangothi P, Tana M, Munster M, Wypkema E, et al. Automated malaria detection by depolarization of laser light. Br J Haematol 1999;104:499-503.

29. Kramer B, Grobusch MP, Suttorp N, Neukammer J, Rinneberg $\mathrm{H}$. Relative frequency of malaria pigment-carrying monocytes of nonimmune and semi-immune patients from flow cytometric depolarized side scatter. Cytometry 2001; 45:133-40.

30. Brown W, Keeney M, Chin-Yee I, Johnson K, Lantis K, Finn W, et al. Validation of body fluid analysis on the Coulter $\mathrm{LH}$ 750. Lab Hematol 2003;9:155-9.

31. Ziebig R, Lun A, Sinha P. Leukocyte counts in cerebrospinal fluid with the automated hematology analyzer CellDyn 3500 and the urine flow cytometer UF-100. Clin Chem 2000; 46:242-7.

32. Soogarun S, Sirimongkolsakul S, Wiwanitkit V, Siritantikorn A, Pimsane K, Srivijarn R. Leukocyte counts in cerebrospinal fluid with the automated hematology analyzer, Technicon $\mathrm{H}^{*}$ 3. Clin Lab 2002;48:623-9.

33. Strik H, Luthe H, Nagel I, Ehrlich B, Bahr M. Automated cerebrospinal fluid cytology: limitations and reasonable applications. Anal Quant Cytol Histol 2005;27:167-73.

34. Terstappen LW, de Grooth BG, Visscher K, van Kouterik FA, Greve J. Four-parameter white blood cell differential counting based on light scattering measurements. Cytometry 1988;9:39-43.

35. Gulati GL, Hyland LJ, Kocher W, Schwarting R. Changes in automated complete blood cell count and differential leukocyte count results induced by storage of blood at room temperature. Arch Pathol Lab Med 2002;126:336-42.

36. Barnes PW, McFadden SL, Machin SJ, Simson E, International consensus group for hematology. The international consensus group for hematology review: suggested criteria for action following automated CBC and WBC differential analysis. Lab Hematol 2005;11:83-90. 